\title{
Delta-slow solution to explain B supergiant stars' winds
}

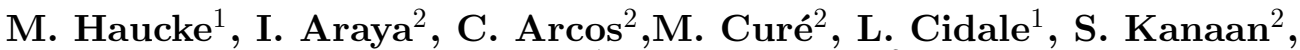 \\ R. Venero ${ }^{1}$ and M. Kraus ${ }^{3}$ \\ ${ }^{1}$ Facultad de Ciencias Astronómicas y Geofísicas, UNLP, Argentina. \\ email: mhaucke@f caglp.unlp.edu.ar \\ ${ }^{2}$ Instituto de Física y Astronomía, UV, Chile. \\ ${ }^{3}$ Astronomický ústav, Akademie věd České Republiky, Ondřejov, Czech Republic.
}

\begin{abstract}
A new radiation-driven wind solution called $\delta$-slow was found by Curé et al. (2011) and it predicts a mass-loss rate and terminal velocity slower than the fast solution (m-CAK, Pauldrach et al. 1986). In this work, we present our first synthetic spectra based on the $\delta$-slow solution for the wind of B supergiant (BSG) stars. We use the output of our hydrodynamical code HYDWIND as input in the radiative transport code FASTWIND (Puls et al. 2005). In order to obtain stellar and wind parameters, we try to reproduce the observed $\mathrm{H} \alpha, \mathrm{H} \beta, \mathrm{H} \gamma, \mathrm{H} \delta$, HeI 4471, HeI 6678 and HeI 4686 lines. The synthetic profiles obtained with the new hydrodynamical solutions are in good agreement with the observations and could give us clues about the parameters involved in the radiation force.
\end{abstract}

Keywords. stars: mass loss, stars: winds, outflows

\section{Introduction}

For O supergiant stars (OSGs) and BSGs, the classical theory of radiation-driven winds predicts that their winds' velocity fields can be fitted by a $\beta$-law, with a typical value of $\beta=0.8-1$. This describes very well the OSGs winds, but for BSGs, observations show values of $\beta>1$, indicating a slower outflowing regime at the base of the wind. To explain this behaviour we present here a preliminary analysis, via a line fitting procedure, of the wind of 3 BSGs (HD 52382 , HD 86440 and HD 91619) using this new $\delta$-slow solution. To fit the $\mathrm{H}$ and He line profiles we used the FASTWIND code, but the input file of the models were done with the code HYDWIND, developed by Curé (2004), which predicts slower and denser winds than the m-CAK theory.

\section{Results}

The parameters obtained from the line fitting for the observed stars are tabulated in Table 1, and the plots are shown in Figure 1.

- The $\delta$-slow solution fits very well the observations, however for HD 91619 we need values of $T_{\text {eff }}$ and $\log g$ higher than the expected for its spectral type (B7).

- For HD 86440 and HD 91619 we obtain a good fit with a $\delta$-slow solution using values of $\delta>0.4$.

\section{Discussion and conclusions}

- The $\delta$-slow solution seems to describe the wind structure of the BSGs with $\beta>1$ at the base of the wind, then it behaves like a $\beta=0.7$ for an intermediate region 
Table 1. Stellar, line force and wind parameters

\begin{tabular}{ccccccccccc}
\hline $\begin{array}{c}\text { STAR } \\
\text { HD }\end{array}$ & $\begin{array}{c}\boldsymbol{T}_{\text {eff }} \\
\mathrm{K}\end{array}$ & $\begin{array}{c}\log \boldsymbol{g} \\
\mathrm{dex}\end{array}$ & $\begin{array}{c}\boldsymbol{v}_{\text {rot }} \\
\mathrm{km} \mathrm{s}^{-1}\end{array}$ & $\begin{array}{c}\boldsymbol{R}_{\star} \\
R_{\odot}\end{array}$ & $\boldsymbol{\alpha}$ & $\boldsymbol{\delta}$ & $\mathbf{k}$ & $\begin{array}{c}\dot{\boldsymbol{M}} \\
10^{-6} \\
M_{\odot} \mathrm{yr}^{-1}\end{array}$ & $\begin{array}{c}\boldsymbol{v}_{\infty} \\
\mathrm{km} \mathrm{s}^{-1}\end{array}$ & $\begin{array}{c}\boldsymbol{v}_{\text {micro }} \\
\mathrm{km} \mathrm{s}^{-1}\end{array}$ \\
\hline 52382 & 19000 & 2.5 & 100 & 52 & 0.52 & 0.39 & 0.21 & 0.47 & 290 & 5 \\
86440 & 15000 & 2.55 & 60 & 62 & 0.52 & 0.43 & 0.32 & 0.15 & 376 & 10 \\
91619 & 19000 & 2.3 & 40 & 71 & 0.52 & 0.44 & 0.142 & 0.5 & 222 & 25 \\
\hline
\end{tabular}

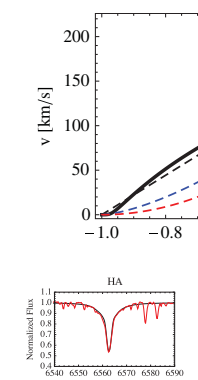

${ }_{\mathrm{H} A \mathrm{~A}]}^{[\mathrm{B}]}$

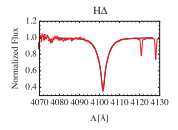

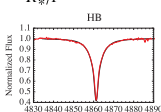

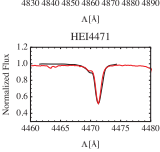

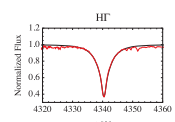

${ }_{\text {HE[ }[6] 78}^{\text {[A] }}$

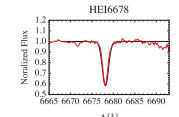

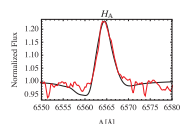

AE1 10678
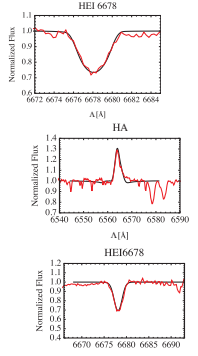
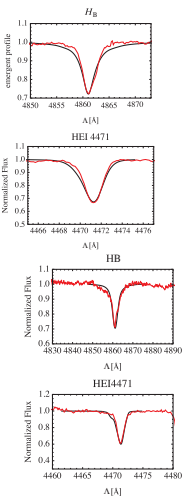

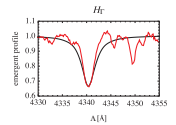

시시
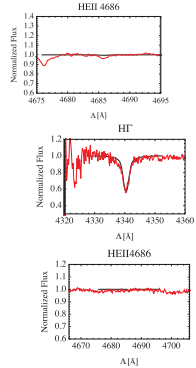

Figure 1. The upper left graphic shows the velocity field of HD 91619. Black solid line is obtained with $\delta$-slow solution. Black, blue and red dashed lines correspond to $\beta$-law with $\beta=1$, 1.5 and 2, respectively. The other panels are the fittings to $\mathrm{H}$ and He lines for HD 52382 (upper right), HD 86440 (bottom left) and HD 91619 (bottom right).

$\left(1.1<r / R_{\star} \lesssim 3.0\right)$, but finally, in the outer part of the wind, it approaches again to $\beta>1$ (see Figure 1). The $\delta$-slow solution reproduces well the observed line profiles.

- HD 52382 is a variable star with no cyclical behaviour (see Morel et al. 2004) and this could be the cause of the differences in the stellar and wind parameters found in the literature, for example, $v_{\infty}=900 \mathrm{~km} \mathrm{~s}^{-1}$ (Howarth et al. 1997) and $\dot{M}=0.33 \times$ $10^{-6} M_{\odot} \mathrm{yr}^{-1}$ (Morel et al. 2004).

- For HD 86440 our results of the $T_{\text {eff }}$ and $\log g$ are in agreement with the same values obtained by Fraser et al. (2010).

- For HD 91619 we have the same "difficulties" as Markova et al. (2008): if we use lower $T_{\text {eff }}$ and lower $\log g$ the absorption component of the synthetic profiles is very intense and does not fit the observed one.

- To reproduce some observations we may need $\delta$ values $>0.4$. These values are greater than $1 / 3$ which is the value expected for a pure $\mathrm{H}$ medium (Puls et al. 2000).

\section{References}

Curé, M. 2004, ApJ 614, 929

Curé, M., Cidale, L., \& Granada, A. 2011, ApJ 737, 18

Fraser, M., Dufton, P. L., Hunter, I., \& Ryans, R. S. I. 2010, MNRAS 404, 1306

Howarth, I. D., Siebert, K. W., Hussain, G. A. J., \& Prinja, R. K. 1997, MNRAS 284, 265

Markova, N., Prinja, R. K., Markov, H., et al. 2008, A\&A 487, 211

Morel, T., Marchenko, S. V., Pati, A. K., et al. 2004, MNRAS 351, 552

Pauldrach, A., Puls, J., \& Kudritzki, R. P. 1986, A\&GA 164, 86

Puls, J., Springmann, U., \& Lennon, M. 2000, A\& $A S$ 141, 23

Puls, J., Urbaneja, M. A., Venero, R., et al. 2005, A\&ऽA 435, 669 